\title{
On the coexistence of ultra-wideband and narrowband radio systems
}

\author{
Ananthram Swami, Brian Sadler, Joi Turner \\ U.S. Army Research Laboratory, Adelphi, MD
}

\begin{abstract}
Ultra-wideband (UWB) signals will encounter many interference sources, primarily from relatively narrowband (NB) systems. In addition, UWB signals will also affect a large number of NB radios; of critical importance is the potential interference with GPS, E-911, and navigation bands, as well as cellular bands. There is a rich and growing literature on UWB radios; however, issues related to interference measurements have only been partially addressed. Here, we assess the interference caused by UWB signals via analysis and simulations. Analytical results include the aggregate effect of spatially distributed UWB radios on a receiver, and theoretical BER expressions.

Keywords - Ultra-wide band systems, interference analysis
\end{abstract}

\section{INTRODUCTION}

There has been a growing interest in ultra-wideband (UWB) radios because of their promise of large bandwidths and co-existence with conventional narrowband systems. Proposed military applications of UWB radios include LPI applications, sensor networks, WLANs for indoor and outdoor command posts, geolocation, RF tagging, inventory control, etc. Typical UWB radios have bandwidths of about $1.6 \mathrm{GHz}$ centered around $2.0 \mathrm{GHz}$; prototype radios with bandwidths of 3-4 GHz, centered at frequencies of 3-4 GHz have been reported. Transmitted powers are typically around $1 \mathrm{nW} / \mathrm{Hz}$. Because of the large bandwidths, and the proposed proliferation, UWB radios will affect a large number of existing narrow-band (NB) systems. It is important to assess the potential interference with GPS, E-911, and navigation bands, as well as cellular bands. Although there is a rich and growing literature on UWB radios, issues related to interference have only been partially addressed, see e.g., [1]-[6].

In order to develop techniques for interference suppression (such as blankers, non-linear pre-processors, etc.), it is important to develop analytical and empirical models of the interference. In this study, we consider the effects of UWB signals on typical NB radios, by adopting appropriate receiver models. The analysis tools are not very different from those used in studying the impact of conventional direct-sequence spread-spectrum and other multiple access signals on classical NB systems.

The theoretical analysis takes into account the waveforms of the interferers (Gaussian monocycles for UWB, typically NB Gaussian processes for NB), the spatial distribution of the sources (density and distances), propagation

The authors are with the U.S. Army Research Laboratory, AMSRLCI-CN, 2800 Powder Mill Road, Adelphi, MD 20783-1197, USA. Email: (aswami,bsadler,jturner)@arl.army.mil. losses, and receiver models (i.e., the nominal signal processing algorithms). The interfering UWB signal structure will cause effects different from that of thermal or broadband noise; in particular, the pulse repetition rate, duty cycles, burst times and specifics of the waveforms play significant roles.

\section{Interference ANALYsis}

Assume that a narrow-band system is operating at a carrier frequency, $f_{c}$ or wavelength $\lambda=c / f_{c}$. We consider the effect of an UWB radio on the NB radio. We do this in two steps: (a) Gross power analysis; (b) detailed waveform analysis. Before proceeding with the analysis, we need to describe the UWB signal.

\section{A. Model for the UWB pulse}

We adopt the usual second-derivative of the Gaussian model for the basic UWB pulse-shape; this leads to a symmetric pulse with an effectively limited temporal duration. Let $p(t)$ denote the pulse-shape, [7], [8]

$$
p(t)=\left[1-2\left(\pi t f_{o}\right)^{2}\right] \exp \left(-\left(\pi t f_{o}\right)^{2}\right)
$$

whose Fourier transform is given by,

$$
P(f)=\frac{2}{\sqrt{\pi f_{o}^{2}}}\left(\frac{f}{f_{o}}\right)^{2} \exp \left(-\frac{f^{2}}{f_{o}^{2}}\right)
$$

which peaks at $f=f_{o}$. The energy in this pulse is $3 / \sqrt{32 \pi f_{o}^{2}}$. Conceptually, a pulse-train (which may be dithered to remove spectral lines, to accommodate user codes, to represent data via pulse-position modulation, etc.) is convolved with the pulse-shaper, so that the power spectrum of the transmitted UWB signal is essentially given by $P^{2}(f)[9]$. The pulse $p(t)$, and $P^{2}(f)$ are shown in Fig. 1; notice that the PSD is skewed. The bandwidth of the transmitted signal is given in Table I. To a first-order approximation, the bandwidth is $2 f_{o}$, and is centered at $f_{o}$.

TABLE I

BANDWIDTh OF UWB SIGNAL USING GAUSSIAN MONOCYCLE: FREQUENCIES NORMALIZED BY $f_{o}$.

\begin{tabular}{|c|c|c|c|}
\hline Attenuation & Low & High & Bandwidth \\
\hline $3 \mathrm{~dB}$ & 0.6169 & 1.4415 & 0.8246 \\
\hline $20 \mathrm{~dB}$ & 0.1955 & 2.2113 & 2.0158 \\
\hline $40 \mathrm{~dB}$ & 0.0607 & 2.7638 & 2.7031 \\
\hline
\end{tabular}



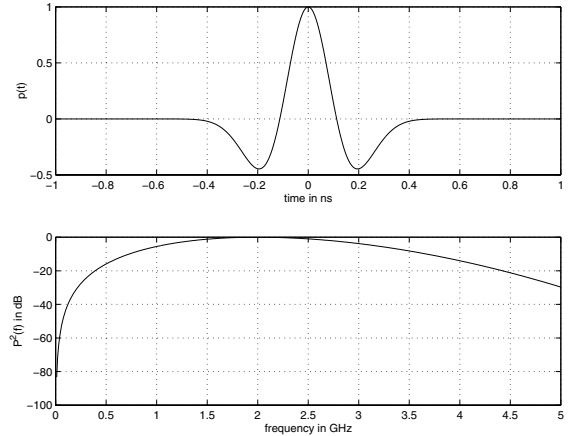

Fig. 1. Gaussian pulse and UWB PSD; $f_{o}=2 \mathrm{GHz}$.

We note from Fig. 1 that the UWB PSD will be basically constant over the bandwidth of a typical narrow-band signal. Consequently, we expect that the effect of the UWB signal is essentially to raise the effective noise floor. If $P_{R}$ denotes the total received power from the UWB signal, then the noise floor will be raised by approximately $P_{R} B W / 2 f_{o}$, where $B W$ is the bandwidth of the NB signal; for a typical wide-band signal with a $300 \mathrm{MHz}$ bandwidth, centered at $f_{c}=2 \mathrm{GHz}$, we have $B W / 2 f_{c}=7.5 \%$. For a more typical narrow-band signal with a $25 \mathrm{KHz}$ bandwidth, centered at $400 \mathrm{MHz}$, we have $B W / 2 f_{c}=6.25 \times 10^{-6} \ll 1$. As with any spread-spectrum type signal, its impact on a narrow band signal decreases with its bandwidth (assuming constant power).

As far as the signal waveform is concerned, the UWB pulse is very impulsive if $B W$ is small; hence, we should expect to see the impulse response of the IF band-pass filter. Depending upon the precise ratios, some ringing may also be evident.

\section{B. Received power}

A gross characterization of the geometric path loss is given by,

$$
P_{R}=P_{o}\left(\frac{d_{o}}{d}\right)^{\beta}
$$

where $P_{R}$ denotes the received power at distance $d$ from the source, $\beta$ is the path loss exponent (typically between 2 and 4 ), and $P_{o}$ is the power measured at distance $d_{o}$ from the transmitter, assuming that the propagation loss from transmitter to reference is also of the form $1 / d^{\beta}$.

In order to assess the aggregate effect of a number of radios (not necessarily UWB), we consider the following scenario. We assume that the receiver uses an omnidirectional antenna. The transmitters all have the same transmit power and are uniformly distributed, with density $\rho$ per square unit, over a concentric ring, centered at the receiver, with inner and outer radii $R_{\min }$ and $R_{\max }$. The transmitted signals are assumed to be independent of each other; additionally it is assumed (although not strictly needed) that all transmitters use the same modulation format. The assumption of equal transmit powers is reasonable in a scheme where power control is difficult. The receiver's basic operation would be to project the received signals onto the signal space which describes the signal of interest. For example, in a conventional narrow-band system, this projection would consist of a band-pass filter (BPF), followed by down-conversion to baseband, lowpass filtering to get rid of the double frequency terms, and matched filtering with the transmit pulse shape.

With $P_{o}$ denoting received power at reference distance $d_{o}$, as described above, the total received power at the center is given by,

$$
\begin{aligned}
P_{R} & =P_{o} d_{o}^{\beta} \rho \int_{R_{\min }}^{R_{\max }} r^{-\beta}(2 \pi r) d r \\
& =P_{o} d_{o}^{\beta} \rho \frac{2 \pi}{\beta-2}\left[R_{\min }^{2-\beta}-R_{\max }^{2-\beta}\right] \\
\lim _{\max } \rightarrow \infty & P_{R}=P_{o}\left(\frac{d_{o}}{R_{\min }}\right)^{\beta} \times \frac{2 \pi R_{\min }^{2} \rho}{\beta-2} .
\end{aligned}
$$

In this equation, the first term describes the received power due to a transmitter at a distance $R_{\min }$; the second term describes the aggregate effect due to all the transmitters. Note that since $\rho$ has dimensions of 1 /area, the second term is dimensionless. The term $\pi R_{\min }^{2} \rho$ represents the effective number of sources within a circle of radius $R_{\min }$; the denominator is related to the path loss exponent. Thus, the aggregate effect is represented by a single transmitter at the minimum distance, $R_{\min }$, whose received power at the reference point is $P_{o}^{\prime}$, given by

$$
P_{o}^{\prime}=P_{o} \frac{2 \pi R_{\min }^{2} \rho}{\beta-2} .
$$

\section{PDF of aggregate noise}

There has been speculation that the aggregate received signal is heavy-tailed. We establish this analytically. In the preceding, we assumed that the transmitters are uniformly distributed; one could also assume that the transmitters are Poisson distributed over the plane. In the latter case, we can use the LePage series theorem [10] in the same manner as in [11]. As $R_{\text {min }} \rightarrow 0$, we can then conclude that the received power is $\alpha$-stable, with characteristic exponent $\alpha=2 / \beta$; we have implicitly assumed that the path loss parameter satisfies $\beta>1$. Thus, the received signal is non-Gaussian with infinite variance. The reader is referred to [10] for details about $\alpha$-stable distributions.

The effects of log-normal shadowing and Rayleigh fading are also easily incorporated into the analysis; the conclusion is again that the aggregate noise is $\alpha$-stable with exponent $\alpha=2 / \beta$. Simulation results in [11] indicate that even with 10 interferers (in a frequency-hopping system), the stable model is a very good approximation. Pedantically, the infinite variance is a consequence of the fact that $R_{\text {min }} \rightarrow 0$. Practically, however, the implication is that the aggregate signal is non-Gaussian. We note that recent reports [6], [12] describe the signal due to a single UWB source at a NB receiver as being much heavier-tailed than the Gaussian. The LePage theorem quantifies this heavytailedness. If the aggregate power is small enough, then this can be lumped with the Gaussian thermal noise. Optimal 
detection in non-Gaussian noise involves non-linear preprocessing to eliminate the deleterious effects of the heavytailed noise. Robust algorithms for this are described in [13], [14], and references therein.

If the duty cycle is small (or the total number of interfering UWB pulses per NB symbol interval is small), a Bernoulli model offers an excellent analytic approximation. If $p$, the probability of a UWB pulse, is small, the normalized kurtosis $\left(6 p^{2}-6 p+1\right) / p(1-p) \approx 1 / p$, indicating that the interference is strongly non-Gaussian. (The nonGaussianity can be exploited to estimate the frequencyselective channel encountered by the UWB signal.)

\section{Waveform Analysis}

In a typical NB radio, using digital modulation, the same symmetric baseband pulse-shape is used at both the transmitter and the receiver, namely the root-raised cosine pulse (RRCP) with transfer function $H_{r c f}(f)$ with nominal bandwidth $W$, roll-off factor or excess bandwidth parameter denoted by $\gamma(0 \leq \gamma \leq 1)$, and overall bandwidth $W(1+\gamma)[15]$.

Taking into account the fact that the front-end bandpass filter and the low-pass filter following the demodulator typically have larger bandwidth than $H_{r c f}(f)$, we note the overall filter for the UWB pulse-train is given by,

$$
\begin{aligned}
G(f) & =\frac{1}{2}\left[P\left(f-f_{c}\right)+P\left(f+f_{c}\right)\right] H_{r c f}(f) \\
& :=\bar{P}(f) H_{r c f}(f)
\end{aligned}
$$

where $P(f)$ is the FT of the Gaussian monocycle [c.f., eqn $(2)]$, and $f_{c}$ is the carrier frequency for the NB system. If the bandwidth of $H_{r c f}(f)$ is very small, then $P(f)$ is essentially flat over the range $\left[f_{c}-W(1+\gamma), f_{c}+W(1+\gamma)\right]$, so that $G(f) \approx P\left(f_{c}\right) H_{r c f}(f)$.

\section{E. Effects on Performance}

Let $x(t)$ denote the received signal, after downconversion to baseband. Consider the usual matched filter plus threshold receiver for BPSK signaling, which is optimal for the AWGN channel. The received signal is

$$
x(t)=A s(t)+w(t)+i(t),
$$

where $s(t)$ is the signal waveform with duration $T, A$ is the unknown amplitude, $w(t)$ is AWGN with two-sided PSD $N_{o} / 2$, and $i(t)$ is the interference. After matched filtering, we have

$$
\begin{aligned}
z & =\int_{0}^{T}[A s(t)+w(t)+i(t)] s(t) d t \\
& =E_{b}+\bar{w}+v .
\end{aligned}
$$

Here $E_{b}$ is the received signal energy per bit, $\bar{w}$ is zero-mean Gaussian, with variance $N_{o} E_{b} / 2$. Assuming that the interfering pulse $i(t)$ is completely contained within the symbol period, and has a relative delay of $\epsilon$; we have

$$
v=\int_{0}^{T} i(t) s(t) d t=\sqrt{E_{p}} \int_{-\infty}^{\infty} \bar{P}(-f) S(f) e^{j 2 \pi f \epsilon} d f
$$

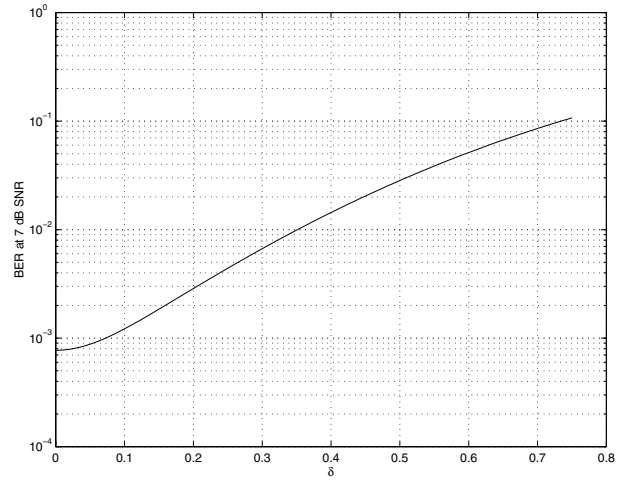

Fig. 2. BER impairment due to UWB pulse

where $\bar{P}(f)$ is defined in $(3)$, and $E_{p}$ is the energy in the received UWB pulse. For a NB $S(f), \bar{P}(f)$ is essentially constant over the bandwidth of $S(f)$; hence,

$$
v \approx \sqrt{E_{p}} P\left(f_{c}\right) s(\epsilon)
$$

Let $s(t)=\sqrt{E_{b}} s_{o}(t)$, so that $s_{o}(t)$ has unit energy; define the SNR impairment factor,

$$
\delta:=\sqrt{\frac{E_{p}}{E_{b}}} P\left(f_{c}\right) s_{o}(\epsilon) .
$$

Then, the bit-error rate (BER) is given by

$$
P_{e}=\frac{1}{4} \operatorname{erfc}\left(\sqrt{\frac{E_{b}}{N_{o}}}(1+\delta)\right)+\frac{1}{4} \operatorname{erfc}\left(\sqrt{\frac{E_{b}}{N_{o}}}(1-\delta)\right) \text {. }
$$

Fig. 2 shows the impairment in BER as a function of $\delta$ for a nominal SNR $\left(E_{b} / N_{o}\right)$ of $7 \mathrm{~dB}$. Fig. 3 shows the extra SNR required to keep the BER constant.

For a RRCP $s_{o}(t)$, it is easy to verify that $\max _{t}\left|s_{o}(t)\right|=$ $1.61 \sqrt{W}$, where $W$ is the nominal bandwidth; thus, $\delta \propto$ $\sqrt{W / f_{o}}$. For $W=50 \mathrm{KHz}, f_{c}=2 \mathrm{GHz}, f_{o}=2 \mathrm{GHz}$, $\delta \approx 5.810^{-3} \sqrt{E_{p} / E_{b}}$. To obtain $\delta=0.1$, we need $E_{p} / E_{b}=$ 300; from Fig. 3, we see that a $3 \mathrm{~dB}$ SNR impairment occurs when $\delta=0.27$; this corresponds to a ratio $E_{p} / E_{b} \approx 2200$. As expected, significant degradation occurs only when the UWB pulse has large energy, since only a small fraction of the UWB energy is passed by the IF filter. As is well known, a more serious problem is saturation due to the large peak power in the UWB pulse (typical numbers are 27 $\mathrm{dB}$ larger than the average power). This suggests the need for high-speed analog blanking circuits in order to cope with dynamic range issues with high-rate A/D convertors. In general, robust signal processing techniques are required throughout the receiver chain.

The BER in (4) is a function of the timing delay $\epsilon$; Fig. 4 displays the average BER (averaged over $\epsilon$ ) as a function of the interference-to-signal ratio (ISR) defined by ISR = $\sqrt{E_{p} / E_{b}} P\left(f_{c}\right)$; the nominal SNR $\left(E_{b} / N_{o}\right)$ was $7 \mathrm{~dB}$.

\section{Simulation Results}

In this section we describe a simulation of the effects of multiple UWB emitters on a narrow band BPSK receiver. 


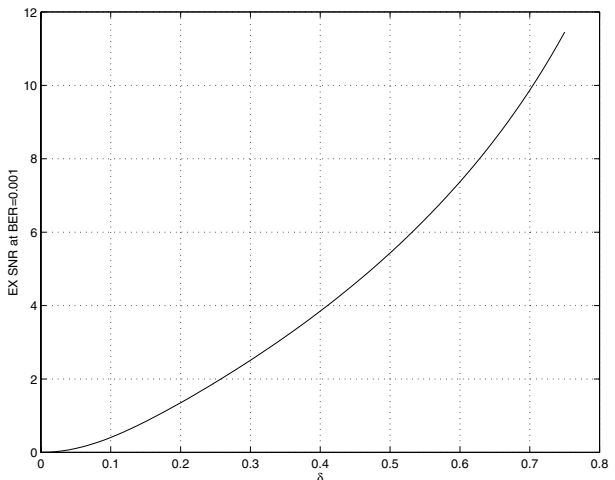

Fig. 3. SNR loss due to UWB pulse

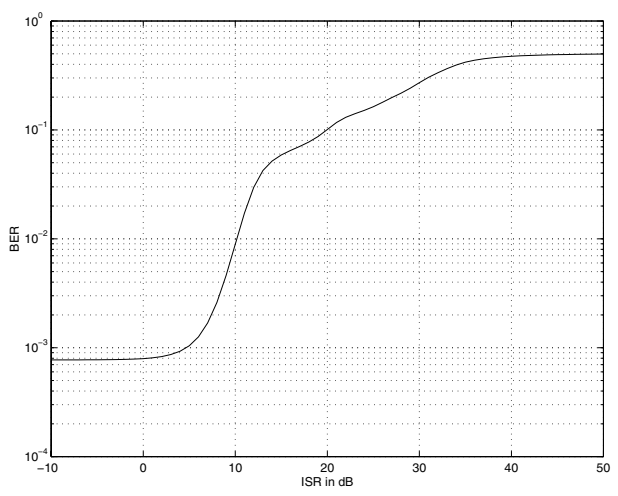

Fig. 4. BER averaged over timing effects

The UWB pulse was modeled as a pure Gaussian pulse, $p(t)=\exp \left(-t^{2} / 2 \sigma^{2}\right)$; this waveform is somewhat different from that described in (1), but the detailed waveform is not important, as is evident from the analysis in Section III. Parameter $\sigma \approx 1 / f_{u w b}$ was adjusted so that the spectral energy is largely confined to the bandwidth $[-1,1] \mathrm{GHz}$; the pulsewidth is about 2 nsec.

The BPSK signal had a rate of $50 \mathrm{Kbps}$, i.e., $T_{b}=40 \mu \mathrm{s}$, and carrier frequency $f_{c}=500 \mathrm{MHz}$. The receiver bandwidth is extremely narrow band with respect to the UWB pulse bandwidth, with an approximate fractional bandwidth of $\left(50 \times 10^{3} / 10^{9}\right) \times 100=0.005 \%$. A RRCP with $\gamma=0.5$ (50\% excess bandwidth) was used.

BER estimates, based on $10^{5}$ BPSK symbols, are shown in Fig. 5. Three cases are shown: AWGN only, AWGN plus a single UWB interferer, and AWGN plus 10 UWB interferers. Each UWB interferer is assumed to have random non-overlapping pulse times, and the pulses are randomly bipolar. The UWB pulse rate is taken to be one fourth the BPSK bit rate. The pulse energy was set to $E_{p}=10^{8}$. This significantly large pulse energy is required due to the very small amount of energy that passes the IF filter of the BPSK system.

\section{ExTENSIONS}

This paper has studied some aspects of the impact of UWB systems on NB systems; the reverse situation has

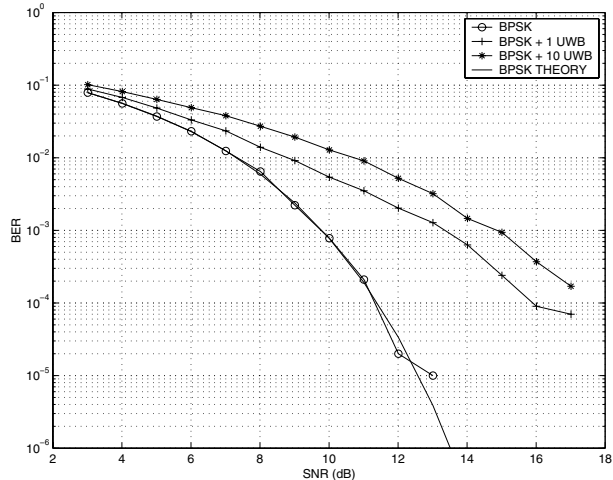

Fig. 5. Performance of BPSK receiver with multiple UWB interferers

not been addressed here, so that 'co-existence' issues have not been fully clarified. We note that the models and analysis implicitly include the effects of multipath. Extensions of current interest include: analysis and simulation of multiple UWB interferers with lognormal shadowing and Rayleigh fading; testing the goodness of fit of $\alpha$ stable models to the aggregate UWB interference; analysis of the impact of blankers and other robust non-linear preprocessors, along the lines described in [13], [14]; and the impact of NB waveforms on UWB receivers.

\section{REFERENCES}

[1] M. Luo, D. Akos, S. Pullen and P. Enge, Potential interference to GPS from UWB transmitters. Test Plan - Ver. 4.5 http://www.ostpxweb.dot.gov/gps/docs/tp_4_5_final.doc

[2] International Ultra Wideband conference, Washington DC, Sept 1999.

[3] Milcom: Session 38, Ultra-Wideband Communications, Proc. IEEE Milcom, Atlantic City, Nov 1999.

[4] Multiple Access Communications Ltd, UK, An investigation into the potential impact of ultra-wideband transmission systems, Feb 2000 .

[5] ONR Ultra wideband Workshop, Berkeley, May 2000 ftp://ftp.onr.navy.mil/PUB/freebej/UWB

[6] NTIA, Assessment of compatibility between ultrawideband devices and selected federal systems, NTIA Publication 01-43, US Dept. of Commerce, Jan 2001.

[7] R.A. Scholtz, "Multiple access with time-hopping impulse modulation", Proc. Milcom, Oct 1993.

[8] M. Win and R.A. Scholtz, "Ultra-wide bandwidth time-hopping spread-spectrum impulse radio for wireless multiple-access communications", IEEE Trans. Commun., 48(4), 679-689, 2000.

[9] M.Z. Win, "Power spectral density of binary digital pulse streams in the presence of independent uniform timing jitter", Proc. Milcom, 1997.

[10] G. Samorodnitsky and M.S. Taqqu, Stable non-Gaussian random processes: stochastic models with infinite variance, Section 1.4, Chapman \& Hall, 1994.

[11] J. Ilow and D. Hatzinakos, "Analytic alpha-stable modeling in a Poisson field of scatterers or interferers", IEEE Trans. Sig. Proc. 46(6), 1601-11, June 1998.

[12] W.A. Kissick, Ed., The temporal and spectral characteristics of ultrawideband signals, NTIA Report 01-383, U.S. Dept. of Commerce, January 2001. Available at www.its.bldrdoc.gov

[13] A. Swami and B. Sadler, "Parameter estimation for linear alphastable processes", IEEE Sig. Proc. Lett., 5(2), 48-50, Feb 1998.

[14] A. Swami, "Non-Gaussian Mixture Models for Detection and Estimation in Heavy-Tailed Noise", Proc. ICASSP'00, vol 6, 380205, June 2000, Istanbul, Turkey.

[15] S. Haykin, Communication Systems, Chapter 7, John Wiley \& Sons, 3rd Edition, 1994. 